\title{
Prevention of Impurity Accumulation in Classical Tokamaks
}

\author{
Tihiro Ohkawa \\ GA Technologies Inc., San Diego, CA 92138
}

( Received November 27, 1984)

Abstract

A new method of impurity control in toroidal devices is proposed. Density inhomogeneity on flux surfaces produced by beam injection or $\mathrm{rf}$ heating creates a poloidal electric field. The resultant impurity ion distribution and orbit leads to the reversal of the impurity inflow.

The definition of the classical tokamaks here is that all transport processes are classical (due to binary collisions including neo-classical or geometrical effects). Three transport time constants are of interest : 1) particle confinement $\tau_{p}, 2$ ) energy confinement time $\tau_{E}$, and 3 ) impurity accumulation time $\tau_{\mathrm{Z}}$. The energy confinement time is shorter than the particle confinement time by the factor $\sqrt{\mathrm{M}_{\mathrm{e}} / \mathrm{M}_{\mathrm{i}}}$, the square root of the electronion mass ratio. The impurity accumulation time is shorter than the energy confinement time by the factor $\mathrm{Z}$ ( charge).

The fact that the impurity accumulation time is the shortest is troublesome. Even if we find a knob to make a tokamak quiescent and classical, it is likely that we would conclude that the discharge is no good because of the impurity accumulation. The knob twisting of experimentalists may be a way to compromise among three time scales by inducing turbulence. Therefore, it is important to find a method to prevent the classic impurity accumulation and then to do the knob twisting in search of a classical tokamak. It may not be a coincidence that impurity problems are severe whenever good confinement is achieved in tokamaks and stellarators.

Several methods have been proposed in the past to reverse the inflow of the impurities. Injection of neutral gas ${ }^{(1,2)}$ changes the circulation of the hydrogenic ions and the flow is reversed by reversing the direction of the frictional force between the impurity ions and hydrogenic ions. Injection of high energy beam ions ${ }^{(3,4)}$ may do the job by imparting momentum on the impurity ions. In this note, a new method that utilizes phenomena already occurring in beam heated tokamaks is proposed.

When neutral beams are injected into a tokamak, some of the resultant fast ions are in trapped orbits. The fraction of ions in trapped orbits depends on the injection angle and 
the penetration depth. The result is that the plasma density becomes inhomogeneous on a flux surface. In order to keep the quasi-neutrality of the plasma, a poloidal electric field develops. In the fluid (collisional) regime, the electric field causes the poloidal inhomogeneity of the impurity density. More specifically, the impurity density becomes higher in the region of smaller major radius.

In the kinetic (long mean-free path) regime, the orbits of impurity ions are modified due to the poloidal electric field. The drift velocity of impurity ions due to Ex $\mathrm{B}$ motion becomes larger than the drift velocity due to $\nabla \mathrm{B}$. In either regime, the net results are to reverse the radial flow of the impurity ions.

We consider the collisional case first. The classical impurity transport is the result of the interaction between the hydrogenic ions and the impurity ions. We consider a steady state in which the force on each species vanishes. The force ${ }^{(5)}$ balance equations in circular tokamaks are given by

$$
\begin{aligned}
& -\frac{\partial p_{i}}{\partial r}+e n_{i} E_{r}+e n_{i} v_{i \theta} B_{\phi}-e n_{i} v_{i \phi} B_{\theta}=0 \\
& -\frac{\partial p_{i}}{r \partial \theta}+e n_{i} E_{\theta}-e n_{i} v_{i r} B_{\phi}-C n_{i} n_{z}\left(v_{i \|}-v_{z \|}\right) \quad \frac{B_{\theta}}{B}=0 \\
& e n_{i} V_{i r} B_{\theta}-C n_{i} n_{z}\left(v_{i / /}-v_{z / /}\right) \quad \frac{B_{\phi}}{B}=0
\end{aligned}
$$

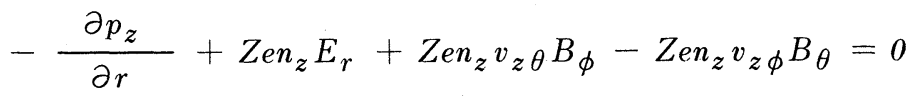

$$
\begin{aligned}
& -\frac{\partial p_{z}}{r \partial \theta}+Z e n_{z} E_{\theta}-e n_{z} v_{z r} B_{\phi}+C n_{i} n_{z}\left(v_{i}\left\|-v_{z}\right\|\right) \cdot \frac{B_{\theta}}{B}=0 \\
& Z_{e n} V_{z r} B_{\theta}+C n_{i} n_{z}\left(v_{i} / / v_{z / /}\right) \quad \frac{B_{\phi}}{B}=0
\end{aligned}
$$

where the subscripts $\mathrm{i}$ and $\mathrm{z}$ denote the hydrogenic and the impurity ion quantities, respectively; the coordinates are $(\mathrm{r}, \theta, \phi) ; \mathrm{E}$ is the electric field; $\mathrm{B}$ is the magnetic field; $\mathrm{v}$ is the 
fluid velocity; $\mathrm{p}$ is the pressure, $\mathrm{n}$ is the density; $\mathrm{Z}$ is the charge of the impurity ion; $\mathrm{C}$ is the coefficient for the frictional force and is given roughly by

$$
C \approx \frac{Z^{2} e^{2}}{\sigma}\left(\frac{m_{i}}{m_{e}}\right)^{1 / 2}
$$

$\sigma$ is the electrical conductivity and $\|$ denotes the components parallel to the magnetic field.

The flow has to be divergence-free to prevent charge accumulation, i. e. :

$$
\frac{\partial}{\partial \theta}\left(n v_{\theta} R\right)=0
$$

where $R$ is the major radius.

By using

$$
v_{\|}=\frac{B_{\theta}}{B} v_{\theta}+\frac{B_{\phi}}{B} v_{\phi}
$$

Eq. (1) and Eq. (4) become

$$
\begin{aligned}
& e n_{i} v_{i \theta} R+\left(-\frac{\partial p_{i}}{\partial r}+e n_{i} E_{r}\right) \frac{R B_{\phi}}{B^{2}}-e n_{i} \frac{B \theta}{B} R v_{i \|}=0 \\
& Z e n_{z} V_{z} R+\left(-\frac{\partial p_{z}}{\partial r}+Z e n_{z} E_{r}\right) \frac{R B_{\phi}}{B^{2}}-Z_{e n_{z}} \frac{B_{\theta}}{B} R v_{z \|}=0
\end{aligned}
$$

Since the first terms are independent of $\theta$, the parallel flow is the result of the $\theta$-dependent parts of the second terms. We assume that the inverse aspect ratio $\varepsilon=\mathrm{r} / \mathrm{R}$ to be small and calculate the parallel velocities to the first order of $\varepsilon$. Also, we use the fact that $\mathrm{B}_{\phi} \propto \mathrm{R}^{-1}$ and $\mathrm{B}_{\theta} \propto \mathrm{R}^{-1}$. We obtain

$$
v_{i \|}-v_{z \|}=2 \varepsilon \cos \theta \frac{B_{\phi}}{B B_{\theta}}\left(-\frac{1}{e n_{i}} \frac{\partial p_{i}}{\partial r}+\frac{1}{Z e n_{z}} \frac{\partial p_{z}}{\partial r}\right)
$$


The radial flux is given by

$$
n_{z} v_{z r}=-\frac{2 \varepsilon \cos \theta C n_{i} n_{z} B_{\phi}^{2}}{Z e B_{\theta}^{2} B^{2}}\left(-\frac{1}{e n_{i}} \frac{\partial p_{i}}{\partial r}+\frac{1}{Z e n_{z}} \frac{\partial p_{z}}{\partial r}\right)
$$

The net flux $\Gamma$ across a flux surface then becomes

$$
\begin{aligned}
\Gamma & \equiv(2 \pi) \quad \int r R n_{z} v_{z r} d \theta \\
& =-\frac{(2 \pi)^{2} \operatorname{Rr} 3 \varepsilon^{2} C B_{\phi}^{2} n_{i} n_{z}}{Z e B_{\theta}^{2} B^{2}}\left(-\frac{1}{e n_{i}} \frac{\partial p_{i}}{\partial r}+\frac{1}{Z e n_{z}} \frac{\partial p_{z}}{\partial r}\right) .
\end{aligned}
$$

The densities and the electrostatic potential can be calculated by assuming the electron distribution. For simplicity, we assume that

$$
-\frac{1}{r} \frac{\partial p_{e}}{\partial \theta}-e n_{e} E_{\theta}=0
$$

and that all temperatures $\mathrm{T}$ are equal. We obtain

$$
\begin{aligned}
& \tilde{n}_{z} \approx \frac{4(Z+1)}{Z^{2}+Z+2} \frac{B_{\phi} C n_{i} n_{z}}{B_{\theta}^{2}}\left(-\frac{1}{e n_{i}} \frac{\partial p_{i}}{\partial r}+\frac{1}{Z e n_{z}} \frac{\partial p_{z}}{\partial r}\right) \varepsilon \sin \theta \\
& \widetilde{n}_{i} \approx-\frac{(Z+1)}{2} \tilde{n}_{z} \\
& \tilde{n}_{e} \approx \frac{(Z-1)}{2} \tilde{n}_{z}
\end{aligned}
$$

They show the up-down asymmetry $(\sin \theta$-dependence $)$.

Next we consider the effects of the trapped beam. We make a simplifying assumption that the beam particles do not interact with background particles collisionally. The only effect is to modify the electron density distribution through the electrostatic potential. The beam particle density $\mathrm{n}_{\mathrm{b}}$ distribution is considered given and is given by 
研究論文 Prevention of Impurity Accumulation in Classical Tokamaks Ohkawa

$$
n_{b}=n_{h}(1+\cos \theta) / 2
$$

Neutrality requires that

$$
n_{e}=n_{b}+n_{i}+Z n_{z}
$$

By denoting the modulated quantity with $*$, we have

$$
n_{e}^{*}=n_{b}^{*}+n_{i}^{*}+Z n_{z}^{*}
$$

The steady state, before we consider the friction between the hydrogenic ions and the impurity ions requires

$$
\begin{aligned}
n_{e}^{*} & =n_{e} e \phi / T \\
n_{i}^{*} & =-n_{i} e \phi / T \\
n_{i}^{*} & =-n_{z} Z e \phi / T
\end{aligned}
$$

where $\phi$ is the electrostatic potential.

The combination of Eqs. (21) and (22) yields

$$
\begin{gathered}
\frac{e \phi}{T} \approx \frac{n_{b}^{*}}{n_{e}\left(1+Z_{e f f}\right)} \\
n_{z}^{*} \approx-\frac{Z n_{z} n_{b}^{*}}{\left(1+Z_{e f f}\right) n_{e}} \\
n_{i}^{*} \approx-\frac{n_{i} n_{b}^{*}}{\left(1+Z_{e f f}\right) n_{e}}
\end{gathered}
$$

where $Z_{\text {eff }}=\left(n_{i}+Z^{2} n_{z}\right) /\left(n_{i}+Z n_{z}\right)$. The impurity ions are concentrated in the inboard side of the torus. 


\section{核融合研究 第 53 巻第 2 号 1985 年 2 月}

The relative velocity in the parallel direction between the hydrogenic ions and impurity ions is proportional to $\left\{-\left[\left(1 / n_{i}\right)\left(\partial p_{i} / \partial r\right)\right]+\left[\left(1 / Z n_{z}\right)\left(\partial p_{z} / \partial r\right)\right]\right\}$ as indicated by Eq. (12). The inspection of Eqs. (24) and (25) shows that the modulated parts of the density do not contribute to the parallel friction. However, the net radial flux is altered because of the density modulation. We obtain

$$
\Gamma=-\frac{(2 \pi)^{2} C R r B_{\phi}^{2} n_{i} n_{z}}{Z e B_{\theta}^{2} B^{2}}\left(-\frac{1}{e n_{i}} \frac{\partial p_{i}}{\partial r}+\frac{1}{Z e n_{z}} \frac{\partial p_{z}}{\partial r}\right)\left(3 \varepsilon^{2}-\frac{Z \varepsilon}{1+Z_{e f f}} \frac{n_{b}}{n_{e}}\right)
$$

The radial flux is reversed when

$$
\frac{n_{b}}{n_{e}}>\frac{3 \varepsilon\left(1+Z_{e f f}\right)}{Z}
$$

The method is more effective on high $\mathrm{Z}$ impurities.

Next we consider the same phenomena kinetically. The orbits of particles are banana orbits in the long mean-free path regime. The drift velocity $v_{d}$ in the vertical direction is given by

$$
\begin{aligned}
v_{i d} & \approx \frac{T}{e B R} \\
v_{z d} & \approx \frac{T}{Z e B R}
\end{aligned}
$$

The radial width of the banana $\Delta$ is given by

$$
\begin{aligned}
& \Delta_{i} \approx \frac{m_{i} v_{i} \sqrt{\varepsilon}}{e B_{\theta}} \\
& \Delta_{z} \approx \frac{m_{i} v_{z} \sqrt{\varepsilon}}{Z e B_{\theta}}
\end{aligned}
$$


The parallel flow $\mathrm{v}_{\|}$is then given by

$$
\begin{aligned}
& v_{i \|} \approx-\frac{\sqrt{\varepsilon}}{n_{i}} \frac{\partial n_{i}}{\partial r} \Delta_{i} v_{i}=\frac{1}{n_{i}} \frac{\partial n_{i}}{\partial r} \frac{T \varepsilon}{e B_{\theta}} \\
& v_{z \|} \approx-\frac{\sqrt{\varepsilon}}{Z n_{z}} \frac{\partial n_{z}}{\partial r} \frac{T \varepsilon}{e B_{\theta}}
\end{aligned}
$$

and the relative flow becomes

$$
v_{i \|}-v_{z / /}=\frac{\varepsilon T}{e B_{\theta}}\left(-\frac{\partial n_{i}}{n_{i} \partial r}+\frac{1}{Z n_{z}} \frac{\partial n_{z}}{\partial r}\right)
$$

The last expression is similar to Eq. (12). The friction displaces the impurity banana and the net radial drift results.

When the beam is introduced, poloidal electric field is produced as shown by Eq. (23). The orbit is modified by the $\mathrm{E} \times \mathrm{B}$ drift.

$$
\begin{aligned}
v_{d z} & =\frac{T}{Z e B R}-\frac{\phi}{B r} \\
& =\frac{T}{Z e B R}\left(1-\frac{R n_{b} Z}{r n_{e}\left(1+Z_{e f f}\right)}\right) .
\end{aligned}
$$

The drift reverses the direction if

$$
\frac{n_{b}}{n_{e}} \frac{Z}{\left(1+Z_{e f f}\right)}>\varepsilon
$$

This is similar to Eq. (27) except for the numerical factor. We assume that $\mathrm{n}_{\mathrm{b}} / \mathrm{n}_{\mathrm{e}}$ is small and the drift velocity of the hydrogenic ions is not reversed. The friction between the ion species displaces the impurity banana in such a way as to have net radial outward drift. When the beam density is much greater than the value given by Eq. (36), the impurity ions become trapped at the inboard side electrostatically. The electrostatic banana orbits move radially outward due to the friction with the parallel flow of the hydrogenic ions. 


\section{核融合研究 第53巻第 2 号 1985 年 2 月}

One might consider the usage of the plasma rotation to produce the poloidal electric field. The centrifugal force on the ions increases the ion density at the outboard side. The resultant density modulation produces the poloidal electric field. However, the impurity ions have greater mass which is roughly proportional to the charge number. The centrifugal force and the electrostatic force oppose each other and the concentration of the impurity ions at the onboard side is not produced.

The above calculations were done for circular cross-section tokamaks. The results are similar for tokamaks with noncircular cross sections. A class of configurations with two trapping regions, i. e. the bean-shaped plasma, opens an interesting possibility. The poloidal electric field may be produced by heating the electrons trapped magnetically at the inboard side. For example, extraordinary ECH heating can be applied to the inboard trapped region. The increase of the trapped electrons and the heating of them produce negative electrostatic potential in the region, which in turn attracts the impurity ions.

\section{参考文献}

1) T. Ohkawa, Kakuyugo-Kenkyu 32,67 (1974).

2) K. Burrell, Phys. Rev. Lett. 4I, 1382 (1978).

3) T. Ohkawa, Kakuyugo-Kenkyu 32, 1-7 (1974).

4) W. M. Stacy Jr., D. J. Sigmar, Nucl. Fusion 19, 1665 (1979).

5) P. Rutherford, Phys. Fluids 17, 1782 (1974). 